\title{
Comparative analysis of three quality of life questionnaires for patients with chronic obstructive pulmonary disease during a rehabilitation program
}

\author{
S. Souto-Camba ${ }^{1,2,3}$, L. Gonzalez-Doniz ${ }^{1,2,3}$, A. López-García ${ }^{1,2,3}$, M. Blanco-Aparicio \\ 4, J.L. Saleta ${ }^{5}$ \\ ${ }^{1}$ Universidade da Coruña, Fisioterapia, A Coruña, Spain; \\ ${ }^{2}$ Grupo de Investigación en Intervención Psicosocial y Rehabilitación Funcional, A Coruña, Spain; \\ ${ }^{3}$ Red Salud, Bienestar Físico y Psicológico, A Coruña, Spain; \\ ${ }^{4}$ Complejo Hospitalario Universitario A Coruña, Servicio de Neumología, A Coruña, Spain; \\ ${ }^{5}$ Complejo Hospitalario Universitario A Coruña, Servicio de Medicina Preventiva, A Coruña, Spain
}

Background: Health-related quality of life (HRQoL) has become an important outcome in respiratory patients. Several specific HRQoL questionnaires have been developed, but they are time-consuming to complete and complex to score.

Purpose: To describe the correlations between the Airways Questionnaire 20 (AQ20), the St. Georges Respiratory Questionnaire (SGRQ), Chronic Respiratory Disease Questionnaire (CRDQ), before and after a rehabilitation program.

Methods: In a quasi-experimental study, 31 patients with moderate-severe COPD (mean age $67 \pm 9$ yr.; FEV1 $34.82 \pm 9.55 \%$ predicted) filled the validated Spanish versions of the SGRQ, CRDQ and AQ20 before and after a rehabilitation program (20 sessions, 7 weeks). Subjects were selected by consecutive sampling in the University Hospital of A Coruña (Spain). Assessments were performed at the same time, under the same conditions by the same examiner. Descriptive analysis was performed. The quantitative data is presented as mean and standard deviation $( \pm S D)$. The Wilcoxon test was used for analyze changes in the HRQoL scores. The relationship between two sets of data was analyzed by Spearman's rank correlation test, and a $p$ value of less than 0.05 was considered to be statically significant.

Results: Before the training program, the AQ20 showed significant correlations with the three components and the total score of the SGRQ $\left(r_{s}=0.4\right.$ to 0.69$)$ as well as with the total score of the CRDQ $\left(r_{s}=-0.35\right)$. The CRDQ components of dyspnea, fatigue and mastery did not achieve significant correlations. After the training program, all subjects improved significantly $(p<0.001)$ their HRQoL in the SGRQ, the CRDQ and the AQ20 total scores $(13.45 \pm 9.9 ;-19.67 \pm 11 ; 3.16 \pm .9$ respectively. Wilcoxon sign-rank test; $p<0.001)$. The correlations between AQ20 and SGRQ and CRDQ were stronger after the training ( $r_{s}=0.53$ to 0.75 and $r_{s}=-0.39$ to 0.64 respectively) and were of significance for all the components of the questionnaires. Also, changes in the AQ20 scores correlated quite well with changes in the other HRQoL questionnaires.

Conclusion(s): The AQ20 has showed responsiveness similar to more complex instruments such as SGRQ and CRDQ and capacity to discriminate changes after a rehabilitation program. At baseline, strength of the correlations where lower than the previously reported in the literature, but after the training the correlations where stronger and similar to another studies.

Implications: Since the best of our knowledge this is the first time that the properties of AQ20 are studied in relation to a rehabilitation program. The AQ20 may be useful to monitor HRQoL in the rehabilitation programs of moderate to severe COPD patients, since it is a short and simple way to discriminate positive changes due to the training.

Keywords: Questionnaires; Quality of Life; Chronic obstructive pulmonary disease

Funding acknowledgements: No funding acknowledgements.

Ethics approval: Ethics Committee of the University of A Coruña (Spain). 